\title{
Fluorescent radiocolloids: are hybrid tracers the future for lymphatic mapping?
}

\author{
Sergi Vidal-Sicart ${ }^{1}$ - Fijs W. B. van Leeuwen ${ }^{2}$ Nynke S. van den Berg ${ }^{2}$ • \\ Renato A. Valdés Olmos ${ }^{2,3}$
}

Received: 1 July 2015 / Accepted: 2 July 2015 /Published online: 22 July 2015

(C) Springer-Verlag Berlin Heidelberg 2015

The metastatic status of regional lymph nodes is one of the most significant prognostic factors in melanoma, breast cancer, and other solid tumours with lymphatic spread. After the seminal description of the modern sentinel lymph node (SLN) concept by Morton et al. [1], there have been a number of studies showing that the status of the SLN constitutes an accurate indicator of the status of the regional nodal basins [2-4].

Biopsy of the SLN has become the standard of care for melanoma and breast cancer patients with non-clinically detectable metastases and has improved the clinical outcome on morbidity and, potentially, mortality [5].

Preoperative identification of the SLNs has been traditionally performed using radiotracer injection, lymphoscintigraphy, and, more recently, single photon emission computed tomography combined with computed tomography (SPECT/CT) [6, 7]. Radiocolloids such as nanocolloid enable a high rate of SLN detection (more than $95 \%$ ), a convenient time window to locate the SLNs, and the possibility to map the lymphatic system preoperatively. Such an anatomical roadmap may be used to plan the optimal approach towards the SLNs

This Editorial Commentary refers to the article http://dx.doi.org/10.1007/ s00259-015-3093-7.

Sergi Vidal-Sicart

svidal@clinic.ub.es

1 Nuclear Medicine Department, Hospital Clínic Barcelona, Barcelona, Spain

2 Interventional Molecular Imaging, Department of Radiology, Leiden University Medical Centre, Leiden, The Netherlands

3 Nuclear Medicine Department, Netherlands Cancer Institute-Antoni van Leeuwenhoek Hospital, Amsterdam, The Netherlands before surgery and, subsequently, to recognize their location intraoperatively [8]. Up to $24 \mathrm{~h}$ after initial administration, the same radiotracers still allow for intraoperative identification with a hand-held gamma probe and/or a portable gamma camera [9].

The ability of radiocolloids to be retained in the SLNs for longer periods of time, in combination with high detection rates, is the reason for the success of this radioguidance approach. However, in spite of a high sensitivity, the radiotracer method has poor spatial resolution. As a result, when the SLNs are located within close proximity of the injection site, the activity emitted from the lymph nodes cannot be distinguished from the background signal (shine-through effect). This effect is particularly problematic in head and neck malignancies [10].

To provide visible feedback during the surgical excision radioguidance can be complemented by the use of vital dyes. The complementary use of blue dye, for instance, allows visual identification of the SLNs throughout the procedure. Thus, a majority of SLN procedures and their guidelines currently advise on the use of radiocolloids in combination with blue dyes [11]. By combining dyes with radiocolloids, the accuracy of SLN detection can be significantly improved $[12,13]$. In this combined approach, the radiocolloids help to determine the initial location of the SLNs (which in part may be located too deep to be initially visible via dye staining), whereas the dye provides the visual feedback on the exact location on the node.

Small organic compounds such as blue dyes are lymphangiographic agents that quickly migrate through the lymphatics, thereby yielding a real-time, but very superficial and short-period (30-45 min), feedback on the lymphatics. Blue dyes are easy to prepare as well as inject, but as a result of their superficial nature and quick diffusion, a percentage of SLNs in expected basins of drainage can be missed. For instance, in 
melanoma, only $79 \%$ of the removed radioactive SLNs are actually blue-stained [14]. The percentage of blue-stained SLNs decreases to $70 \%$ in breast cancer [15]. Also, to guide SLN removal in aberrant drainage, blue dyes have limitations, and, for instance, outside the axilla in breast cancer, only $30 \%$ of the internal mammary radioactive SLNs stains blue [16]) and $47 \%$ in other non-axillary basins [17]. Similar to blue dyes, the use of single fluorescent dyes such as indocyanine green (ICG) has shown to slightly improve the detection sensitivity and in depth view; however, they still suffer from the same intrinsic limitations, which are all related to the use of light. This issue was evaluated in a recent study in 52 melanoma patients, where the SLN identification rate were $96.2 \%$ for radiotracer, $59.6 \%$ for blue dye, and $88.5 \%$ for ICG [18].

As a result, the small dyes are most useful during the visualization of lymph nodes located in superficial tissues (i.e. melanoma, breast cancer, penile, and vulvar cancers). However, even here visualization of the SLNs is difficult in overweight patients as in those cases fat tissue completely surrounds the SLNs, thereby attenuating the signal $[19,20]$.

In a prospective study of patients with melanomas of the trunk or extremities, Stoffels et al. reported that ICG provided inferior performance compared to $99 \mathrm{mTc}$-nanocolloid in defining lymphatic basins at risk for metastases before surgery. With the standard technique using 99mTc-radiocolloid, they detected and excised 147 SLNs in 80 patients with melanoma. In only $21 \%$ of patients ( 17 of 80 ) was the SLN detectable by ICG before skin incision [21]. These findings are consistent with other studies showing that although fluorescence imaging allowed for intraoperative identification of the SLNs, the limited degree of tissue penetration does not allow for accurate SLN mapping prior to surgery $[18,20]$.

Moreover, these findings once more underline that radiocolloids remain the gold standard for preoperative SLN mapping via, e.g., lymphoscintigraphy in patients with melanoma and other cancers. This approach also enables the use of SPECT/CT in patients with malignancies draining to complex anatomical areas, such as the head and neck.

To take advantage of the excellent properties of radiotracers for preoperative SLN mapping, while complementing these features with optical detection of the exact same SLNs, the hybrid SLN tracer, ICG-99mTc-nanocolloid, combining radioactivity and fluorescence in one tracer was developed [22]. During the development process it was also clearly demonstrated that binding ICG to $99 \mathrm{mTc}-\mathrm{HSA}$ provided inferior results compared to ICG-99mTc-nanocolloid, meaning the size of the colloid is instrumental for the success of this tracer [23].

ICG- ${ }^{99 \mathrm{~m}} \mathrm{Tc}$-nanocolloid has, to date, been the most widely used hybrid tracer for SLN biopsy [24]. The rational for a hybrid tracer rests on the combination of the fluorescent and radioactive signatures in one compound; this may improve logistics in daily clinical practice, as no additional injections during surgery are needed to allow for optical SLN identification. More interesting is that the properties of this hybrid radiotracer permits its use in both 1- and 2-day protocols.

Also important is that ICG-99mTc-nanocolloid can preoperatively be used for lymphatic mapping in a manner identical to the one previously described for $99 \mathrm{mTc}$-nanocolloid. In a recent comparison study it was assessed that ICG- ${ }^{99 \mathrm{~m}} \mathrm{Tc}$ nanocolloid shows a drainage pattern similar to its parental compound ${ }^{99 \mathrm{~m}} \mathrm{Tc}$-nanocolloid [25]. In addition to preoperative imaging, this same tracer enabled intraoperative fluorescence guidance to the exact SLNs identified via preoperative imaging. The feasibility of this approach has been demonstrated in a variety of cancers, such as melanoma, breast cancer, prostate cancer, penile cancer, vulvar cancer, and oral cavity cancer [26-31]. The hybrid guidance was considered particularly helpful in areas where no vital blue dye is habitually used, with a complex anatomy and/or with near-injection site SNs. An example of such an area wherein this approach has previously been evaluated is the head and neck [32].

More recently, the hybrid approach has been validated in melanoma. In this evaluation optical SLN identification rate, enabled by the fluorescent signature of the hybrid tracer was found to be superior to the one achieved with just vital blue dye. For example, in melanoma it was found intraoperatively that $97 \%$ of SNs could be visualized with fluorescence imaging, whereas only $62 \%$ of these nodes had stained blue. Interestingly, in all the reported studies, ex vivo examination of the excised SLNs confirmed the combined presence of a radioactive and fluorescent signature [26].

In this issue of Eur J Nucl Med Molecular Imaging, Stoffels et al. report a randomized prospective study comprising 40 patients with stages Ib and II melanoma, squamous cell, Merkel cell, and sweat gland carcinoma in the head and neck region. Of this group, 20 patients received a derivative of the previously reported ICG-99mTc-nanocolloid tracer, while the rest of the patients were given the standard $99 \mathrm{mTc}-$ nanocolloid. Primary study end points were the number of excised SLN and the duration of surgical intervention. It was concluded that the use of ICG-99mTc-nanocolloid for SLN biopsy is feasible in patients with head and neck cancer. Further, the authors state that by combining fluorescence and radioactivity in one single tracer, discrepancies between the two imaging modalities used for SLN localization are less likely to occur [33].

Together with preoperative lymphoscintigraphy and SPECT/CT imaging, Stoffels et al. performed intraoperative fluorescence imaging to determine whether the lymphatic pathways and SLN could be visualized via fluorescence imaging through the intact skin. In $20 \%$ of the patients $(4 / 20$; cohort A) this was possible. These findings are comparable to previously reported studies [20]. In cases when the fluorescent signal was not visible the handheld gamma probe was used to determine the point of incision and further preparation. The 
time for SLN biopsy was not significantly different between these groups (36 vs. $45 \mathrm{~min}$ ). All procedures were performed 19-27 h after hybrid tracer injection. From their study the authors conclude that the hybrid tracer-based approach is feasible and provides a fully integrated pre- and intraoperative approach.

Some aspects from this paper, however, remain a matter of discussion.

For instance, the authors mention that they expected a limited concordance between the two methods because of the challenging identification of deep SLNs due to the limited tissue penetration of the fluorescence signal. This is an understandable starting point when ICG and ${ }^{99 \mathrm{~m}}$ Tc-nanocolloid are used separately, but is a surprising and unexplained starting point of a study where a hybrid tracer is used that has a proven concordance in findings.

It is actually not clear why the authors took this starting point for their study. This may be partially because of the set end point of number of excised SLNs. If they would have specified here that their aim was to look specifically at the number of excised SLNs via fluorescence guidance only, this point might have been proven valid. However, since they used the combined approach, in which the radioguidance complements the fluorescence guidance (and vice versa) this statement remains confusing. Throughout the paper, the authors base their findings on a per-patient basis rather than on an SLN-based basis. The best approach for reporting the results can be discussed.

The randomized prospective setup of the study is very well chosen. Yet, in their comparative study the authors have been using different doses for ${ }^{99 \mathrm{~m}} \mathrm{Tc}$-nanocolloid and ICG- ${ }^{99 \mathrm{~m}} \mathrm{Tc}$ nanocolloid. In the latter group, the amount of nanocolloid is twice as high as in the second group. This means that in their comparison they do not only look at different features, but also at different particle densities, which may severely have influenced their findings. In fact, it may have lead to the identification of more lymph nodes considered as SLNs. This aspect has been previously evaluated when the particle density was changed in prostate cancer patients [34, 35].

Further in the paper, in $18 / 20$ patients that received ${ }^{99 \mathrm{~m}} \mathrm{Tc}$ nanocolloid preoperatively, SLNs could be identified via lymphoscintigraphy vs. $100 \%$ in the patients $(20 / 20)$ that received the hybrid tracer ICG- ${ }^{99 \mathrm{~m}} \mathrm{Tc}$-nanocolloid. In four patients SLNs were transcutaneously visible. However, the authors do not report on the number, nor on the location of these SLNs. Intraoperatively, in cohort B ( ${ }^{99 \mathrm{~m}}$ Tc-nanocolloid) in 19/ 20 patients (95\%), a total of 34 SNs could be identified and removed via the radioguided approach. In cohort $\mathrm{A}$ (ICG- ${ }^{99 \mathrm{~m}}$ Tc-nanocolloid), a total of 36 SLNs were removed in all patients. Of these 36 removed SLNs, six were removed exclusively on fluorescence guidance, which led the investigators to conclude that with the hybrid tracer more SLNs are removed, which might lead to side effects. It is not reported where these SLNs were located and if, for example, they might have been part of a cluster or if these nodes were located at a very different location from the preoperatively identified SLNs in the respective two patients. If these weren't radioactive, this could be explained as described by the authors by some free ICG that might have dissolved from the hybrid tracer resulting in the finding of nodes that are solely fluorescent. Unfortunately, an ex vivo evaluation on the excised tissue to determine whether or not both signatures could be detected was not performed. Such an evaluation is recommended before one may conclude that one of the two signatures is indeed not present. On the other hand, a different formulation for $99 \mathrm{mTc}$-nanocolloid was used, namely Nanotop, which can influence the binding efficacy to ICG and may, therefore, have caused this possible dissemination. A more logical explanation might, therefore, be the time interval between injection and operation (19-27 h) where the radioactive signal can no longer be detected due to decay. Unfortunately, the injected dose has not been specified in the paper.

During the propagation of new tracers that have been introduced in the clinic it would be best if some form of standardization was maintained. Although Stoffels et al. did a noteworthy job, due to the aforementioned differences it is difficult to compare their findings with previous studies using the same tracer in the head and neck area. Probably controlled multicenter trials with a standardized setup will provide outcome and at the same time will contribute to introduce this new technology into the clinical routine.

In this context, a hybrid tracer integrating radioactivity and fluorescence in one signature represents the basis of an original approach that not only combines the best of both diagnostic worlds for the sentinel node procedure, but also constitutes a working model aimed at extending the frontiers of nuclear medicine.

\section{References}

1. Morton DL, Wen DR, Wong JH, Economou JS, Cagle LA, Storm FK, et al. Technical details of intraoperative lymphatic mapping for early stage melanoma. Arch Surg. 1992;127:392-9.

2. Ball CG, Sutherland F, Kirkpatrick AW, Dixon E, MacLean AR, Mack LA, et al. Dramatic innovations in modern surgical subspecialties. Can J Surg. 2010;53:335-41.

3. Krag DN, Anderson SJ, Julian TB, Brown AM, Harlow SP, Costantino JP, et al. Sentinel lymph node resection compared with conventional axillary lymph node dissection in clinically node negative patients with breast cancer: overall survival findings from the NSABPB-32 randomised phase 3 trial. Lancet Oncol. 2010;11: 927-33.

4. Morton DL, Thompson JF, Cochran AJ, Mozzillo N, Nieweg OE, Roses DF, et al. Final trial report of sentinel-node biopsy versus nodal observation in melanoma. N Engl J Med. 2014;370:599-609.

5. Leong SP, Zuber M, Ferris RL, Kitagawa Y, Cabanas R, Levenback $\mathrm{C}$, et al. Impact of nodal status and tumor burden in sentinel lymph 
nodes on the clinical outcomes of cancer patients. J Surg Oncol. 2011;103:518-30.

6. Moncayo VM, Aarsvold JN, Alazraki NP. Lymphoscintigraphy and Sentinel Nodes. J Nucl Med. 2015;56:901-7.

7. Vidal-Sicart S, Brouwer OR, Valdés-Olmos RA. Evaluation of the sentinel lymph node combining SPECT/CT with the planar image and its importance for the surgical act. Rev Esp Med Nucl. 2011;30: $331-7$.

8. Mariani G, Bruselli L, Kuwert T, Kim EE, Flotats A, Israel O, et al. A review on the clinical uses of SPECT/CT. Eur J Nucl Med Mol Imaging. 2010;37:1959-85.

9. Giammarile F, Alazraki N, Aarsvold JN, Audisio RA, Glass E, Grant SF, et al. The EANM and SNMMI practice guideline for lymphoscintigraphy and sentinel node localization in breast cancer. Eur J Nucl Med Mol Imaging. 2013;40:1932-47.

10. Frontado LM, Brouwer OR, van den Berg NS, Mathéron HM, Vidal-Sicart S, van Leeuwen FW, et al. Added value of the hybrid tracer indocyanine green-99mTc-nanocolloid for sentinel node biopsy in a series of patients with different lymphatic drainage patterns. Rev Esp Med Nucl Imagen Mol. 2013;32:227-33.

11. Chakera AH, Hesse B, Burak Z, Ballinger JR, Britten A, Caracò C, et al. European Association of Nuclear Medicine-European Organisation for Research and. EANM-EORTC general recommendations for sentinel node diagnostics in melanoma. Eur J Nucl Med Mol Imaging. 2009;36:1713-42.

12. Mariani G, Erba P, Manca G, Villa G, Gipponi M, Boni G, et al. Radioguided sentinel lymph node biopsy in patients with malignant cutaneous melanoma: the nuclear medicine contribution. J Surg Oncol. 2004;85:141-51.

13. Mariani G, Erba P, Villa G, Gipponi M, Manca G, Boni G, et al. Lymphoscintigraphic and intraoperative detection of the sentinel lymph node in breast cancer patients: the nuclear medicine perspective. J Surg Oncol. 2004;85:112-22.

14. Kapteijn BA, Nieweg OE, Muller SH, Liem IH, Hoefnagel CA, Rutgers EJ. Validation of gamma probe detection of the sentinel node in melanoma. J Nucl Med. 1997;38:362-6.

15. Doting MH, Jansen L, Nieweg OE, Piers DA, Tiebosch AT, Koops HS, et al. Lymphatic mapping with intralesional tracer administration in breast carcinoma patients. Cancer. 2000;88:2546-52.

16. Estourgie SH, Tanis PJ, Nieweg OE, Valdés Olmos RA, Rutgers EJ, Kroon BB. Should the hunt for internal mammary chain sentinel nodes begin? An evaluation of 150 breast cancer patients. Ann Surg Oncol. 2003;10:935-41.

17. van Rijk MC, Tanis PJ, Nieweg OE, Olmos RA, Rutgers EJ, Hoefnagel CA, et al. Clinical implications of sentinel nodes outside the axilla and internal mammary chain in patients with breast cancer. J Surg Oncol. 2006;94:281-6.

18. Cloyd JM, Wapnir IL, Read BM, Swetter S, Greco RS. Indocyanine green and fluorescence lymphangiography for sentinel lymph node identification in cutaneous melanoma. J Surg Oncol. 2014;110: 888-92.

19. Lerman H, Lievshitz G, Zak O, Metser U, Schneebaum S, EvenSapir E. Improved sentinel node identification by SPECT/CT in overweight patients with breast cancer. J Nucl Med. 2007;48:201-6.

20. Crane LM, Themelis G, Arts HJ, Buddingh KT, Brouwers AH, Ntziachristos V, et al. Intraoperative near-infrared fluorescence imaging for sentinel lymph node detection in vulvar cancer: first clinical results. Gynecol Oncol. 2011;120:291-5.

21. Stoffels I, Dissemond J, Pöppel T, Schadendorf D, Klode J. Intraoperative fluorescence imaging for sentinel lymph node detection: prospective clinical trial to compare the usefulness of indocyanine green vs technetium Tc $99 \mathrm{~m}$ for identification of sentinel lymph nodes. JAMA Surg. 2015. doi:10.1001/jamasurg.2014. 3502.
22. Buckle T, Chin PT, van Leeuwen FW. (Non-targeted) radioactive/ fluorescent nanoparticles and their potential in combined pre- and intraoperative imaging during sentinel lymph node resection. Nanotechnology. 2010;21:482001.

23. van Leeuwen AC, Buckle T, Bendle G, Vermeeren L, Valdés Olmos $\mathrm{R}$, van der Poel HG, et al. Tracer-cocktail injections for combined pre- and intraoperative multimodal imaging of lymph nodes in a spontaneous mouse prostate tumor model. J Biomed Opt. 2011;16: 016004 .

24. van Leeuwen FW, Hardwick JC, van Erkel AR. Luminescencebased Imaging. Approaches in the field of interventional molecular imaging. Radiology. 2015;276:12-29.

25. Brouwer OR, Buckle T, Vermeeren L, Klop WM, Balm AJ, van der Poel HG, et al. Comparing the hybrid fluorescent-radioactive tracer indocyanine green-99mTc-nanocolloid with 99mTc-nano- colloid for sentinel node identification: a validation study using lymphoscintigraphy and SPECT/CT. J Nucl Med. 2012;53:1034-40.

26. van den Berg NS, Brouwer OR, Schaafsma BE, Mathéron HM, Klop WMC, Balm AJM, et al. Multimodal surgical guidance during sentinel node biopsy for melanoma: Combined gamma tracing and fluorescence imaging of the sentinel node through use of the hybrid tracer indocyanine green-technetium-99m-nanocolloid". Radiology. 2015;275:521-9.

27. Schaafsma BE, Verbeek FP, Rietbergen DD, van der Hiel B, van der Vorst JR, Liefers GJ, et al. Clinical trial of combined radio- and fluorescence-guided sentinel lymph node biopsy in breast cancer. Br J Surg. 2013;100:1037-44.

28. van der Poel HG, Buckle T, Brouwer OR, Valdés Olmos RA, van Leeuwen FW. Intraoperative laparoscopic fluorescence guidance to the sentinel lymph node in prostate cancer patients: clinical proof of concept of an integrated functional imaging approach using a multimodal tracer. Eur Urol. 2011;60:826-33.

29. Brouwer OR, van den Berg NS, Mathéron HM, van der Poel HG, van Rhijn BW, Bex A, et al. A hybrid radioactive and fluorescent tracer for sentinel node biopsy in penile carcinoma as a potential replacement for blue dye. Eur Urol. 2014;65:600-9.

30. Mathéron HM, van den Berg NS, Brouwer OR, Kleinjan GH, van Driel WJ, Trum JW, et al. Multimodal surgical guidance towards the sentinel node in vulvar cancer. Gynecol Oncol. 2013;131:720-5.

31. Borbón-Arce M, Brouwer OR, van den Berg NS, Mathéron H, Klop WM, Balm AJ, et al. An innovative multimodality approach for sentinel node mapping and biopsy in head and neck malignancies. Rev Esp Med Nucl Imagen Mol. 2014;33:274-9.

32. van den Berg NS, Brouwer OR, Klop WM, Karakullukcu B, Zuur CL, Tan IB, et al. Concomitant radio- and fluorescence-guided sentinel lymph node biopsy in squamous cell carcinoma of the oral cavity using ICG-(99m)Tc-nanocolloid. Eur J Nucl Med Mol Imaging. 2012;39:1128-36.

33. Stoffels I, Leyh J, Pöppel T, Schadendorf D, Klode J. Evaluation of a radioactive and fluorescent hybrid tracer for sentinel lymph node biopsy in head and neck malignancies: prospective randomized clinical trial to compare ICG- $(99 \mathrm{~m}) \mathrm{Tc}$-nanocolloid hybrid tracer versus (99m)Tc-nanocolloid. Eur J Nucl Med Mol Imaging. 2015.

34. Vermeeren L, Muller SH, Meinhardt W, Valdés Olmos RA. Optimizing the colloid particle concentration for improved preoperative and intraoperative image-guided detection of sentinel nodes in prostate cancer. Eur J Nucl Med Mol Imaging. 2010;37:132834.

35. KleinJan GH, van den Berg NS, Brouwer OR, de Jong J, Acar C, Wit EM, et al. Optimisation of fluorescence guidance during robotassisted laparoscopic sentinel node biopsy for prostate cancer. Eur Urol. 2014;66:991-8. 